index •comunicación | no 11(2) 2021 | Páginas 59-79

E-ISSN: 2174-1859 | ISSN: 2444-3239 | Depósito Legal: M-19965-2015

Recibido el 17_01_2021 | Aceptado el 27_04_2021 | Publicado el 15_07_2021

\title{
DEPORTE Y ESPECTÁCULO EN \\ LA NARRATIVA DE LOS 'E-SPORTS': EL CASO DE 'LEAGUE OF LEGENDS'
}

\section{SPORT AND SHOW IN THE NARRATIVE OF E-SPORT: THE CASE OF 'LEAGUE OF LEGENDS'}

https://doi.org/10.33732/ixc/11/02Deport

\author{
Diego Martín Muñoz \\ Universidad Pontificia de Salamanca \\ diegomartinmunoz690@gmail.com \\ https://orcid.org/0000-0002-1633-7420 \\ Luis Miguel Pedrero Esteban \\ Universidad Nebrija \\ Impedreroes@gmail.com \\ https://orcid.org/0000-0003-4949-2360
}


Resumen: Los deportes electrónicos se han consolidado en el mercado del ocio y entretenimiento digital con cifras de seguidores, facturación y publicidad similares a las de los tradicionales deportes masivos. Además de fomentar la fantasía individual sin limitaciones espacio-temporales como sucede con los videojuegos, los deportes electrónicos se articulan sobre estrategias discursivas y técnicas que amplían el vínculo emocional entre el jugador, el deporte y la audiencia. Este artículo identifica y describe la evolución de los videojuegos a lo largo de su historia, la conversión de muchos de ellos en deportes electrónicos clarificando los elementos que hacen que una disciplina como un videojuego pueda convertirse en un espectáculo-, y se centra en la propuesta lúdica de los deportes electrónicos a partir de un estudio de caso sobre League of Legends, el título más popular a nivel mundial, cuya singular estrategia narrativa le ha convertido en un híbrido de juego, deporte y espectáculo.

Palabras clave: videojuego; narrativa; conexión; deporte electrónico; tecnología.

Abstract: e-sports have stablished themselves in the leisure and entertainment digital market with similar number of followers, turnover and advertising to those of traditional mass sports. In addition to fostering individual fantasy without space-time limitations as it happens with video games, esports are articulated on discursive strategies and techniques that expand the emotional bond between the player, the sport and the audience. This article identifies and describes the evolution of video games throughout their history, the conversion of many of them into e-sports -clarifying the elements that make a discipline such us a videogame becomes a spectacle-, and focuses on the ludic proporsal of e-sports from a case study on League of Legends, the world's most popular title, whose unique narrative strategy has made it a game, sport and spectacle hybrid.

Keywords: Videogame; Narrative; Bond; e-sport; Technology. 


\section{Introducción}

La irrupción de Internet y la normalización global de las redes y dispositivos de acceso al entorno digital han provocado cambios irreversibles sobre los hábitos entretenimiento. El consumo que se hacía de los videojuegos ha cambiado drásticamente: desde el disfrute personal de un niño jugando al Super Mario Bros, hasta compartir tus partidas de Fortnite a través de Twitch.tv, o incluso, llegar a presenciar el último mundial de League of Legends. Y es que los videojuegos cada vez están más presentes en nuestras vidas, prueba de ello es el informe de la Asociación Española del Videojuego (2019), que cuantifica la cantidad de usuarios de esta disciplina en quince millones de personas en el territorio español. Además, también hace una clasificación de los seguidores de los deportes electrónicos, situándolos en casi los tres millones de personas (Asociación Española de Videojuegos, 2019). Mientras que en Europa, se calcula que unos doscientos cincuenta millones de personas son jugadores de videojuegos - el 50\% de la población-y algo más de 35 millones serían seguidores habituales de deportes electrónicos, según la Europe’s Video Games Industry (ISFE, 2020).

Figura 1. Crecimiento de la audiencia de e-sports a lo largo de los años
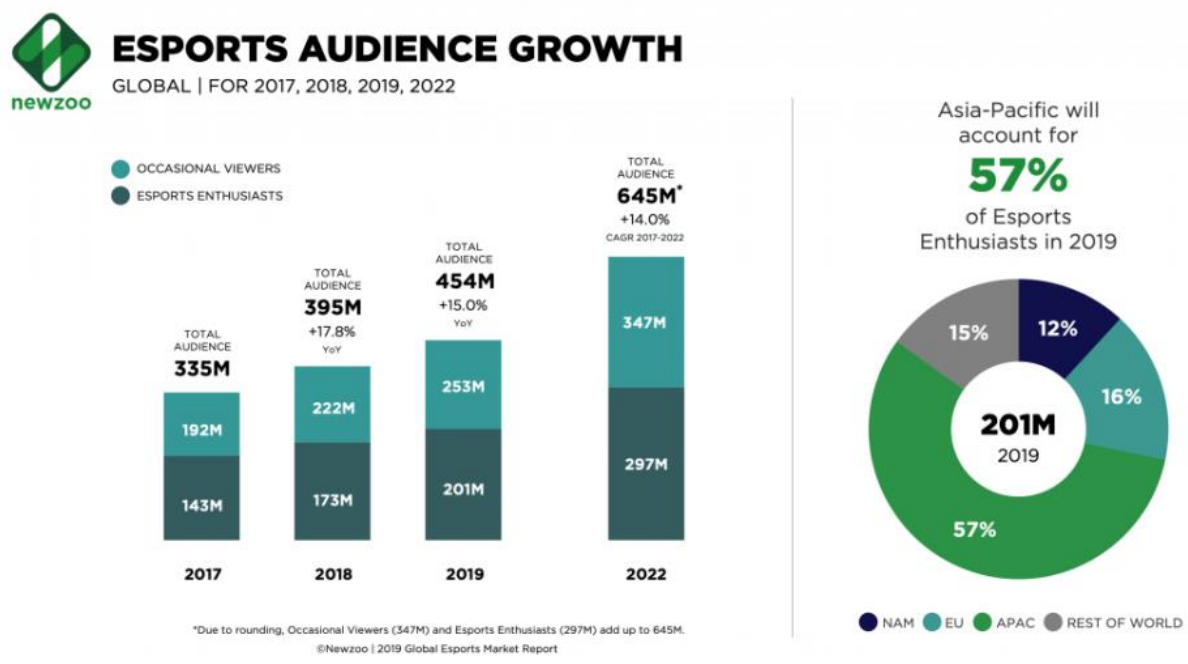

Fuente: Newzoo.

Si extrapolamos estos datos a nivel mundial, y según la consultora especializada Newzoo, en su informe anual, habría más de quinientos millones de personas en el mundo que podrían considerarse consumidores de e-sports 
(Newzoo, 2020). Ante la relevancia de esta forma de entretenimiento, este artículo quiere poner de manifiesto la relación existente entre un deporte electrónico, su videojuego y los usuarios de ambos, como una forma de retroalimentación en el contenido generado en cualquiera de los ámbitos ya citados. Para ello, se realiza un estudio de caso del videojuego League of Legends.

\section{Metodología}

Esta investigación pretende identificar la especificidad ludonarrativa de los deportes electrónicos como relatos que amplían la dimensión individual de los videojuegos y se expanden hacia la social de los deportes tradicionales, en cuanto que ambas prácticas se han convertido en espectáculos de masas. Si bien no puede negarse la naturaleza del videojuego como texto complejo y multiforme, su infraestructura sistémica, la flexibilidad de su composición textual y el valor procedimental de algunos de sus contenidos permiten aproximaciones desde diferentes campos de estudio (Alonso Martínez et al., 2020). Para reconocer las variables que caracterizan las retransmisiones de videojuegos y e-sports, e identificar los elementos que singularizan la narración de los deportes electrónicos, el artículo se centra en un estudio de caso (Hammersley, 1986; Hernández-Sampieri et al., 2014; López-Barajas \& Montoya, 1995; Yin, 1989), que examina League of Legends, el título más popular en el mundo con ocho millones de jugadores simultáneos diarios (Marca Esports, 2019), desde la metodología del análisis textual propuesta por Cassetti y di Chio (1996) para el análisis fílmico.

La metodología del análisis textual consta de cuatro procedimientos generales: un proceso inicial de descomposición de las diversas partes del texto en unidades más pequeñas; un posterior análisis de esas partes; la recomposición de los valores semánticos derivados en las fases de enumeración, ordenamiento, reagrupamiento y modelización; y una comprensión final del fenómeno. En el caso de League of Legends, se trata de un videojuego del género MOBA - Multiplayer Online Battle Arena-, en el que dos equipos de cinco jugadores, apoyados en un personaje con habilidades concretas, tienen como objetivo destruir la base del equipo enemigo con distintas estrategias y enfrentamientos. El videojuego tiene lugar en un mapa cerrado que conecta las dos bases a través de tres carriles custodiados por estructuras defensivas. Este videojuego alcanzó tanto éxito tras su publicación en 2009 que su empresa fundadora, Riot Games, organiza de forma anual un evento mundial que congrega a los mejores equipos y jugadores del mundo. Desde entonces, las audiencias que se han ido generando en torno a esta competición han ido creciendo de manera exponencial. Un ejemplo de ello son los datos cosechados por este $e$-sport en el último torneo mundial, organizado 
en el año 2019 en varias capitales europeas —a pesar de que en el año 2020 también se celebraron los correspondientes mundiales, su impacto se vio condicionado por la pandemia de la COVID 19-. La final, celebrada en París, fue retransmitida en diecinueve idiomas y a través de más de treinta plataformas. El pico de audiencia se cifró en cuarenta y cuatro millones de espectadores únicos, mientras la audiencia media por minuto sobrepasó los veinte millones. En total, y durante todo el evento, los datos recogidos por Riot Games superaron los mil millones de horas visualizadas (Lolesports, 2019).

\section{La transformación de los videojuegos en deportes electrónicos}

El uso de los videojuegos como recursos para el ocio y el entretenimiento comienza a mediados del siglo XX; en aquel momento la sociedad sufría aún las consecuencias de una Segunda Guerra Mundial convertida en la más cruenta de las contiendas bélicas hasta entonces, en gran parte por el desarrollo tecnológico. La creación de radares, por ejemplo, fue una de las principales herramientas utilizadas por ambos bandos y tendría una gran influencia en la industria de los videojuegos, ya que muchos de ellos copiarían su interfaz (Quiroga, 2018). Además, la posterior carrera espacial entre los principales bloques económicos que lideraban el orden mundial, marcaría la temática de los videojuegos venideros (Documanía, 2014). Toda aquella expectación impulsó las inversiones de las grandes universidades, donde la carrera aeroespacial pasó a convertirse en una de las principales aspiraciones de los estudiantes. En ese contexto, en el Instituto Tecnológico de Massachusetts (MIT) se realizaron numerosos avances científicos y se gestaron las bases de lo que hoy conocemos como la Administración Nacional de Aeronáutica y el Espacio (NASA). Además, y sobre todo, se presentó el nuevo ordenador creado por la compañía americana Digital Equipment Corporation (DEC): se denominó PDP-1 y poseía una pantalla CRT Type 30. Para demostrar su potencia, Steve Rusell, Wayne Witaenem y Martín Graetzz, estudiantes universitarios, diseñaron una herramienta interactiva que permitía explotar las prestaciones del rutilante nuevo equipo. Así nació Spacewar!, un videojuego desarrollado con un fin no lúdico, sino enfocado a resaltar el potencial interactivo del PDP1: mediante dos naves llamadas 'cuña' y 'aguja', y sobre un mapa que representaba el espacio exterior, los jugadores debían esquivar asteroides, estrellas y cometas. Ambos vehículos tenían como principal objetivo la destrucción del otro (Scolari, 2013), una temática que evidenciaba la influencia que por entonces generaba la carrera espacial en la sociedad. 
Hasta ahora, estas herramientas no tenían una función lúdica ni se habían creado con ese propósito, pero tras la demostración de Steve Rusell en el MIT, muchas universidades del país se hicieron con la tecnología necesaria para replicar el PDP-1 y el Spacewar! en sus instalaciones; no solo eso: en 1971 verían la luz las dos primeras máquinas recreativas arcade de la historia: Galaxy Game y Computer Space (Kent, 2001).

La primera fue inventada por Bill Pitts y Huggh Tuck. Se trataba de una versión de Spacewar! que cabe considerar como el primer videojuego con propósito comercial: los jugadores pagaban diez dólares por una partida o veinticinco por disfrutar de tres. Además, Pitts y Tuck lograron modificar la máquina arcade para acceder a una experiencia multijugador y lograr así una mayor amortización. La segunda, Computer Space, fue creada por Nolan Bushnell y Ted Dabney, quienes habían sido contratados por Bill Nutting para crear una máquina arcade que se pudiera fabricar en masa; el resultado fue Computer Space, un videojuego que consistía en controlar un cohete y disparar contra dos platillos volantes que controlaba la inteligencia artificial de la máquina. A pesar de sus esfuerzos, las ventas no reportaron a la empresa la rentabilidad esperada, en parte porque su dificultad suponía un hándicap para quienes buscaban un entretenimiento más simple (Velasco, 2011).

Pese a ello, Bushnell y Dabney descubrieron el potencial del sector y decidieron reintentarlo con la fundación de la empresa de videojuegos Atari. Su primer movimiento fue inspeccionar lo que había hasta ese momento en el mercado: Bushnell acudió a la demostración de la Magnavox Odyssey, la primera consola de la historia creada por Ralph Baer a finales de los 60 (aunque no se comercializó hasta 1972). Bushnell disfrutó de la selección de videojuegos que ofrecían y se fijó en uno: el juego de tenis. Junto a Ted Dabney y Allan Alcorn desarrollaron el histórico Pong, cuyo caudal comercial se demostró tras ser probado ante el público en un bar de California y recaudar cuarenta dólares diarios, todo un éxito. Gracias a este impulso, Atari vendió sus primeras once unidades, lo que le permitió ampliar sus instalaciones para hacer frente a un nuevo pedido de cincuenta. Tal fue su aceptación que con los encargos de máquinas Pong no lograron dar abasto, y tras varias tentativas frustradas, optaron por externalizar el proceso de producción y fabricar 2.500 unidades en 1973 y 8.000 al año siguiente. Con ello, Atari, acaba de crear la industria de los videojuegos. A partir de este momento, los videojuegos, que habían nacido con una función orientada al entretenimiento individual, comenzaron a disfrutarse como un ocio compartido y colectivo a través de torneos y competiciones en torno a su título. Estas expresiones serán conocidas como e-sport o deportes electrónicos (Martín Muñoz \& Pedrero Esteban, 2019). 
La primera manifestación de esta nueva disciplina tiene que ver con Spacewar! Tras su creación en el MIT, en 1972 un grupo de alumnos organizó en la universidad de Stanford un torneo sobre este videojuego entre los estudiantes del campus; el ganador se llevó una suscripción a la revista Rolling Stone. La competición no era muy distinta al juego original: se enfrentaban dos jugadores entre sí, cada uno de ellos manejaba una nave espacial con cohetes y ganaba el primero que destruía la nave del oponente (Taylor, 2015). Este torneo, a pesar de no poseer las cualidades necesarias para ser considerado un deporte electrónico, sí puede catalogarse como un primer paso hacia los $e$-sports.

La década de los setenta alumbró numerosos títulos y novedosos aparatos electrónicos, videoconsolas y ordenadores que, cada año, aumentaban las cualidades técnicas de los lanzamientos que salían al mercado. Todos ellos destinados al entretenimiento individual: un jugador con un mando que vive una historia que él mismo va construyendo y en la que se integra a nivel competitivo y emocional: «se trata de que los jugadores y fans estén dentro de las historias, y eso es un viaje de inmersión, un viaje mental», describe la profesora Belén Mainer (Berzosa, 2017: 75).

A pesar de ello, tuvieron que transcurrir diez años hasta que se volvió a organizar algo cercano al concepto e-sport: fue en1980, en un campeonato centrado en el videojuego Space Invaders creado años antes por Atari. Al constatar su éxito, la empresa, decidió organizar un torneo para encontrar al mejor jugador del cartucho. Para ello, la empresa de Bushnell creó un evento en diferentes regiones del país que tenía como objetivo la clasificación para la gran final en la ciudad de Nueva York, a la que llegaron a acudir más de diez mil personas. El torneo consistía en obtener la máxima puntuación eliminando oleadas de alienígenas con el cañón láser. La ganadora fue Rebeca Heinmann, quien logró batir los 110.000 puntos y superó con creces los resultados alcanzados hasta la fecha.

La industria de los videojuegos continuó lanzando nuevos títulos, consolas e incluso ordenadores -equipos en los que trataría de incrementar su alcance - con tal intensidad que terminó por saturar el mercado: la calidad de los títulos fue reduciéndose y la falta de compatibilidad entre las consolas hacía prácticamente imposible que el consumidor pudiese adquirirlas todas, o al menos las necesarias para que fueran rentables para las empresas. A principios de los años ochenta, se produjo una crisis que motivó el cierre de muchas empresas del sector y que también afectó a los deportes electrónicos: no se organizó ningún evento de e-sports hasta 1990, cuando la compañía japonesa Nintendo promovió la Nintendo World Championship para impulsar el 
lanzamiento de su consola NES (Nintendo Entertainment System). El torneo, organizado por la revista perteneciente a la marca, discurrió por distintas ciudades del continente americano bajo el nombre Nintendo Power Fest, y se celebraba durante setenta y dos horas en cada localidad. La empresa japonesa logró así reunir a los treinta mejores jugadores en un gran evento en Hollywood, en los estudios de Universal. La final consistía en tres videojuegos: Super Mario Bros, Rad Racer y Tetris. Para jugar, los aspirantes disponían de seis minutos y veintiún segundos: comenzaban con Super Mario Bros, en el que debían conseguir cincuenta monedas; más tarde pasaban al Rad Racer, un juego de carreras en el que tenían que intentar dar una vuelta a un circuito lo más rápido posible; y terminaban, con el tiempo restante de las otras dos pruebas, intentando conseguir la máxima puntuación posible en Tetris. Los resultados de los tres juegos se acumulaban para la alcanzar la puntuación final.

La Nintendo World Championship resultó un éxito debido a la gran acogida de la NES, convertida en la principal baza para relanzar la industria y acabar con la crisis de 1985. En los años noventa la fiebre por los videojuegos y las consolas se multiplicó, y eso también repercutió en la categoría de los deportes electrónicos, cuya identidad se reforzaría gracias a un nuevo evento organizado en 1997. Red Annihilation fue un torneo que versaba sobre el videojuego Quake, título del género shooter en primera persona, en el que participaron dos mil personas en línea y en el que se clasificaron los dieciséis mejores jugadores. La final se celebró de manera presencial y el ganador se llevó un Ferrari 328 GTS y un premio de 5.000 dólares. Esta competición poseía características propias de los actuales deportes electrónicos y, sobre todo, un rasgo que aún no se había materializado: la competición online. Dos años más tarde se empezó a acuñar el término e-sport: lo hizo por primera vez el periodista Matt Bettington en una nota de prensa desde el sitio especializado en noticias sobre videojuegos, Eurogamers, en la que, al presentar la Asociación de Jugadores en Línea (OGA), comparó a los deportes electrónicos con los tradicionales (Taylor, 2015).

Con el cambio de siglo, se vivió uno de los primeros booms en la industria de los e-sports, al menos en el continente asiático, donde surgieron las primeras ligas de videojuegos como Starcraft. La On Game Net Starleague comenzó su andadura en el año 2001 y duró hasta 2013. Pero sería en 2009 cuando tuvo lugar el gran detonante de la industria con el lanzamiento de League of Legends, desarrollado por Riot Games, que dos años más tarde organizó un evento mundial para enfrentar a los mejores jugadores y nombrar a los campeones del mundo. Si bien apenas mil personas disfrutaron de aquel evento, celebrado en la feria sueca Dreamhack, ayudó a popularizar el videojuego y a darlo a conocer a millones de personas. Tanto, que Riot Games volvió a organizar al año 
siguiente un nuevo evento con sede en Los Ángeles: los premios ascendieron entonces a dos millones de euros y la audiencia total llegó a los nueve millones de personas en su transmisión a través de Internet. Desde entonces, el crecimiento que ha experimentado League of Legends ha sido exponencial: ya no solo es una cita anual y global entre los mejores equipos, ahora hay ligas regionales por todos los continentes en las que se disputan las plazas que dan acceso a la gran cita mundial (Iwerks, 2019).

El evento ha cobrado tal magnitud que las ceremonias de apertura constituyen un espectáculo televisivo comparable al de los grandes deportes tradicionales, como la final de la Superbowl. Con la introducción de la realidad aumentada, confluyen espectáculos virtuales y reales sobre el escenario que aportan una narrativa distinta (Pérez Irigoyen, 2018). La final enfrenta a los dos mejores equipos del mundo en un formato al mejor de cinco mapas; es decir, se juegan cinco partidas de League of Legends y el primer equipo que logra vencer en tres, se alza con el título de campeón. En los últimos años, y contando con la afición del sudeste asiático - la más importante del evento-, se ha conseguido superar la cifra de doscientos millones de personas visualizando simultáneamente la final (Esports Charts, 2018). Fue en 2018, y aunque la cifra resulte estimativa debido a la fórmula de conteo en las plataformas orientales de streaming, los datos no hacen más que incrementarse año tras año también en las regiones occidentales, tanto en Europa como en Estados Unidos (Mellado, 2018).

Desde la primera aparición de los videojuegos y su naturaleza de entretenimiento individual, hasta los grandes espectáculos de masas contemporáneos de los e-sports, se han ido sucediendo varios acontecimientos que han ido dotando a la disciplina de elementos específicos y diferenciales. Por ello, al distinguir entre videojuegos y deportes electrónicos conviene aclarar varios aspectos. En primer lugar, los deportes electrónicos no son videojuegos de deporte, al menos no todos; la relación entre 'deporte' y 'electrónico' se alcanza con la competición entre jugadores a través de dispositivos conectados -ordenador, consola o smartphone- En segundo lugar, no todos los videojuegos pertenecen o pueden pertenecer a la categoría de e-sport: que un videojuego permita a los usuarios competir con otros jugadores no significa que se convierta automáticamente en un deporte electrónico. Cabe entonces formular una definición que abarque las peculiaridades del fenómeno: un $e$ sport es una competición de videojuegos a nivel profesional y en un formato organizado (un torneo o una liga) con un objetivo específico (ganar un título de campeón o un premio en metálico) y una clara distinción entre los jugadores y equipos que compiten entre sí (Martín Muñoz \& Pedrero Esteban, 
2019). Aunque estos eventos se celebran habitualmente de forma presencial ante el público, también se difunden por diferentes canales y tecnologías, y sus derechos de retransmisión se comercializan de forma análoga a los grandes eventos de ocio y entretenimiento televisivo.

\section{Variables narrativas y técnicas en la retransmisión de videojuegos}

Así como las retransmisiones deportivas de radio y televisión se han difundido a lo largo de los años desde diferentes tecnologías de distribución - hertziana analógica, hertziana digital o TDT, cable, satélite y a través de Internet-, la señal de los deportes electrónicos siempre se ha recibido online, mediante un streaming de contenido multimedia al que el usuario accede desde cualquier dispositivo conectado a la red y visualiza al tiempo que se descarga. Se trata de un sistema preferente en la dieta de consumo digital de las generaciones más jóvenes de usuarios - aunque se extiende poco a poco a los públicos de todas las edades (Europa Press, 2019) -, razón que explica las estrategias de la industria tradicional para incorporarse a los nuevos entornos mediante plataformas específicas de vídeo y audio bajo demanda capaces de competir con las ofertas servicios como Netflix (Neira, 2020).

En el ámbito de los videojuegos, el servicio de referencia es Twitch, lanzado en 2011 como un subproducto del portal de videojuegos Justin.tv especializado en $e$-sports y adquirido por la todopoderosa Amazon en 2014. En el cómputo de las interacciones que se realizan durante un minuto en Internet, Twitch superó en 2020 el millón doscientas mil visualizaciones, frente a las algo más de novecientas mil de 2018, el primer año en que se recogieron en la infografía de la consultora Lori Lewis (Lewis, 2020). De hecho, en los últimos meses, el portal ha visto aumentado todos sus registros debido al confinamiento sanitario por la COVID-19. La herramienta de Amazon ha promediado más de dos millones de espectadores, más horas vistas y hasta más horas en directo de los generadores de contenido, con respecto a los datos cosechados por la plataforma durante la primera semana de Marzo, donde la empresa de Amazon establece la división del inicio de la pandemia (Antón, 2020). Aunque Twitch no es una plataforma solo dirigida a videojuegos o deportes electrónicos, la mayoría del contenido que se emite a través de esta plataforma pertenece a esa temática. Además, y aunque con menor impacto hasta la fecha, también ofrecen retransmisiones de deportes electrónicos YouTube, Dailymotion o el servicio over the top (OTT) de deportes de pago DAZN.

El streaming es una de las grandes peculiaridades de los deportes electrónicos y también esencial para entender su funcionamiento. Se opone a la programación televisiva tradicional (lineal) porque brinda un consumo a la 
carta de lo que el usuario desea ver en cada instante. Lo único necesario es registrarse en una cuenta que permite compartir el contenido a través de la red, así como un dispositivo conectado dotado de pantalla (ordenador, tableta o smartphone). Una vez dentro, las posibilidades son infinitas: sistema de alertas personalizado, plantillas que modifican el aspecto del contenido audiovisual para adaptarlo al videojuego, facilidades de interacción con el espectador, que incluso puede elegir la música de fondo... Las opciones de una emisión en directo, a través de una plataforma especializada, son muy numerosas, y cada una es representativa del producto que se quiere mostrar (Taylor, 2018).

Pese a la variedad de streamings en la oferta de Twitch, las emisiones en directo se dividen en dos variantes, al menos las que corresponden a videojuegos: emisiones particulares, que cabe categorizar como creaciones de contenido; y generales, a las que se pueden denominar deportes electrónicos. Las primeras siguen un sencillo patrón de realización que incluye una pantalla general con el contenido principal (normalmente un videojuego); una pequeña ventana situada abajo, a la izquierda o derecha dependiendo de la disposición del contenido, con un primer plano del responsable de la emisión (streamer); y una plantilla gráfica con información complementaria: nombre o apodo del locutor, publicidad y equipo al que pertenece, si este fuera el caso.

En cambio, las emisiones generales que se corresponden con e-sports (siempre que la organización esté emitiendo un torneo de videojuegos) se presentan más organizadas porque su realización corre a cargo de una empresa especializada y, por ello, se enriquecen con gran repertorio técnico y expresivo de elementos: diferentes cámaras, estudio de realización, intervención de varios presentadores, iluminación, piezas audiovisuales editadas con antelación... Es decir, una plasmación asimilable a la de una emisión deportiva en televisión. Esta es la variante dominante en las retransmisiones de e-sports: así ocurre en España con la Liga de Videojuegos Profesional (LVP), cuyos derechos gestiona Mediapro, y en Europa con la League European Championship (LEC) competición de League of Legends que organiza Riot Games como la compañía diseñadora del videojuego.

Dependiendo del título, la distribución de elementos de una retransmisión se organiza de una u otra manera con el fin de no obstaculizar el desarrollo de la acción. En la imagen 1 se observa cómo el streamer sitúa su imagen abajo a la derecha debido a la disposición del juego, manteniendo la visibilidad de la marca para la que trabaja y la de los anuncios que se superponen sobre la animación. Por lo general, las emisiones particulares contienen menos elementos, aunque hay creadores de contenido que invierten muchos recursos en mejorar su producto y por ello incluyen en sus 
retransmisiones realizaciones multicámara, focos e incluso disponen de estudios de grabación.

Imagen 1. Ibai Llanos, creador de contenido, haciendo streaming

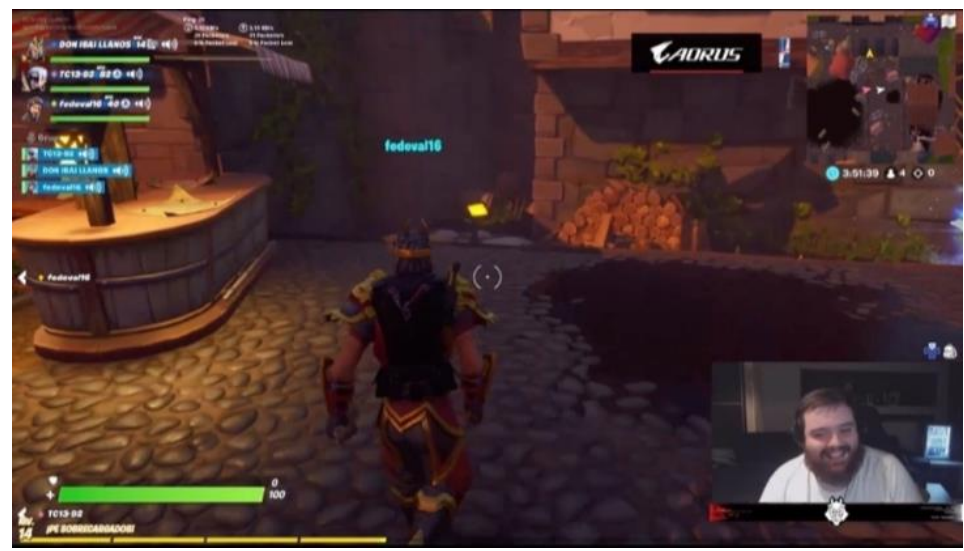

Fuente: Twitch.tv/ibai.

En la imagen 2, la retransmisión de Riot Games a través de Twitch del videojuego League of Legends permite identificar varios elementos. El más importante es el contenido principal, el videojuego, que ocupa la mayor parte de la imagen, pero también hay dos cámaras donde se muestran las reacciones de los jugadores y varios marcadores. El principal, arriba, refleja el desarrollo de la partida, y otro, secundario y más detallado, muestra las estadísticas de los personajes del videojuego de forma individual. A lo largo de la partida aparecen pantallas con estadísticas secundarias, en este caso, aparece a la izquierda de la pantalla.

Imagen 2. Fnatic vs G2 esports. Jornada siete en League European Championship

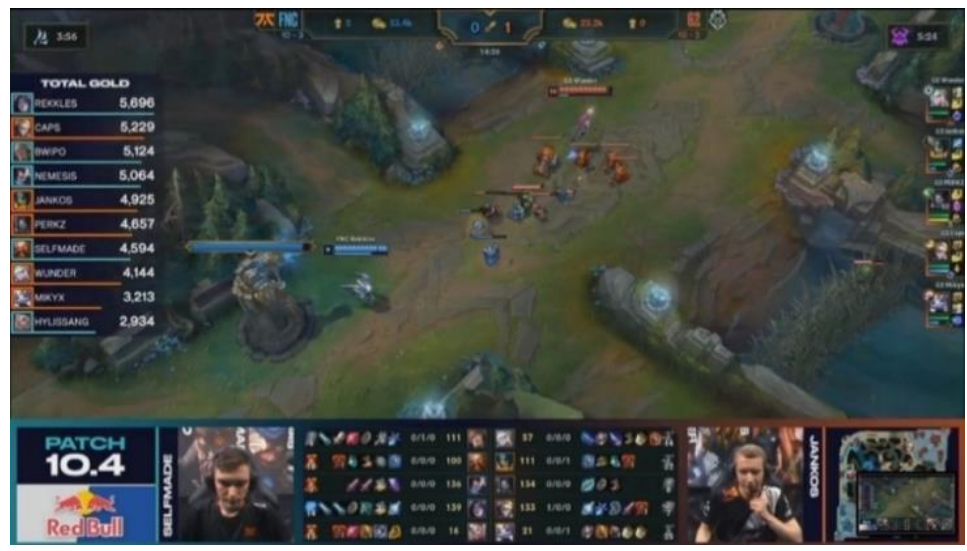

Fuente: Twitch.tv/lvpes. 
Todo ello está apoyado por la narración que llevan a cabo los que se conocen como casters (comentaristas), quienes cuentan el devenir de la partida apoyándose en tecnicismos propios del videojuego o incluso de las redes sociales, pues se trata de entornos muy correlacionados (Carrillo Vera, 2016). Las estadísticas e infografía introducen nuevas perspectivas de la partida (un recurso que en las retransmisiones de fútbol por televisión también va ganando peso gracias a las técnicas de animación e incluso de realidad virtual). Como novedad, desde la temporada 2020 se introdujo en la -entonces'Superliga Orange' la JCAM para conectar con los estadios de los equipos en tiempo real.

\section{La implicación de los usuarios en la narrativa de los e-sports}

Aunque no todos los usuarios de un videojuego compiten en su correspondiente $e$-sport - la gran mayoría no lo hacen-, en los campeonatos que se organizan en torno a él, sí resulta relevante para los creadores, desarrolladores y marcas que se genere una masa de espectadores interesados en el seguimiento de las ligas y torneos, como ocurre en las competiciones de los deportes tradicionales. De manera periódica, y normalmente en momentos en los que la oferta de competiciones de videojuegos es muy alta - como las fases finales de una liga regional o la fase de grupos de un torneo mundial一, Riot Games organiza misiones para todos los usuarios de League of Legends que consisten en visualizar partidas a través de las plataformas proporcionadas para ello a través de las cuales los usuarios pueden conseguir recompensas que, o bien no lograrían por otra vía, o solo conseguirían aportando dinero real (García, 2019).

Como un reflejo más del proceso de convergencia mediática analizado por Henry Jenkins (2008), el videojuego permite apelar de forma directa al usuario para la ampliación de contenidos. En el caso de League of Legends, esa estrategia se ha materializado a través de un sistema de recompensas añadido a las misiones: su funcionamiento resulta similar, pero en lugar de alcanzar objetivos establecidos, los popularmente denominados drops se activan ante determinadas circunstancias de una partida. Así, cuando en una retransmisión de un mapa de League of Legends se logra una remontada, o hay alguna jugada espectacular (entre otras posibilidades), el sistema arroja recompensas a los espectadores que en ese instante visualicen el encuentro y que son canjeables dentro del videojuego. También se introducen en este sistema de recompensas, descuentos y ofertas en el material o la indumentaria del equipo protagonista (Díaz, 2020). 
La búsqueda de la implicación de los usuarios como espectadores que reciben estímulos para después jugar a un videojuego conforma una novedosa narrativa que no solo fomenta el consumo pasivo de los e-sports, sino también, $\mathrm{y}$, sobre todo, la participación activa y el aumento en el volumen de los seguidores de este fenómeno. Con esta pretensión se diseñó también la herramienta 'SLO Superfantasy', otro formato de competición estilo 'comunio' que, junto al visionado de partidas, le propone al espectador un mini-juego con el que pasar a formar parte de la competición. La propia Riot Games ha integrado esta iniciativa - con el nombre de Fantasy League- en el campeonato europeo de League of Legends. Su funcionamiento consiste en involucrar al espectador en los partidos de la liga a través de una competición de cromos. El juego simula que el usuario es un entrenador y tiene que hacer una alineación con los jugadores de los que dispone en esa semana, que acabarán puntuando según su actuación en la liga real de acuerdo con estadísticas muy concretas. La recompensa habitual es el desbloqueo de regalos que solo se podrían conseguir introduciendo dinero real en el videojuego (Liga de Videojuegos Profesional, 2020).

Imagen 3. Disposición del Fantasy en la pantalla del usuario

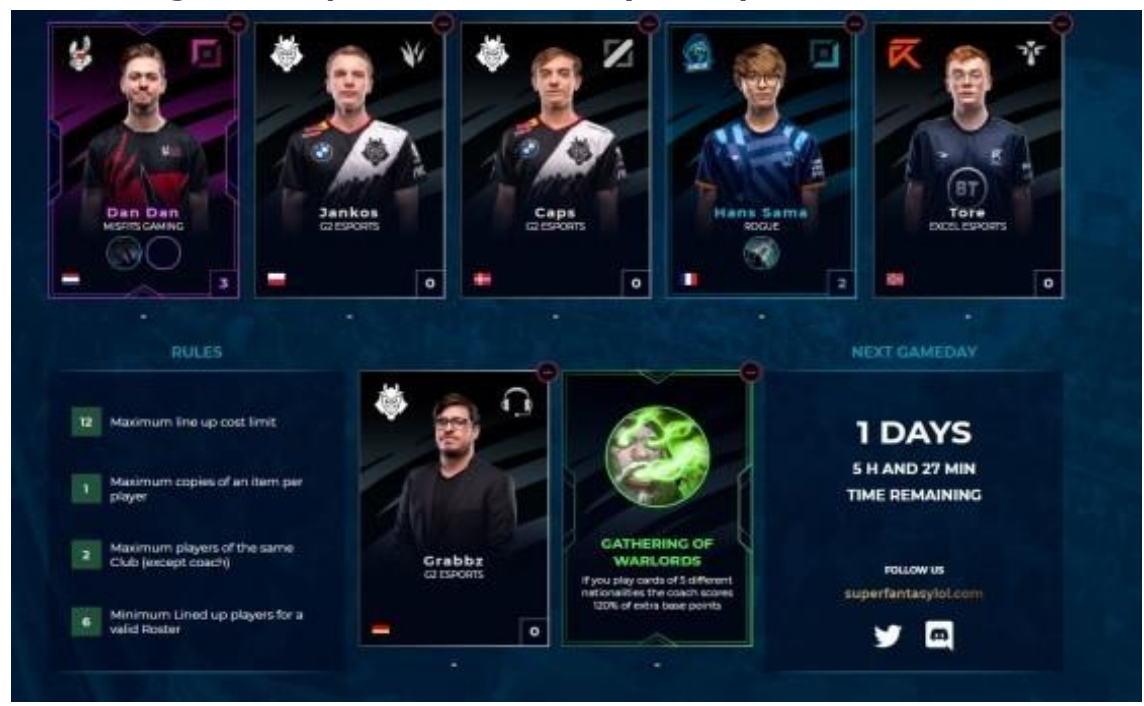

Fuente: Millenium.gg.

Además, la herramienta permite crear una liga privada con los participantes que el usuario quiera, o bien ingresar en un torneo público organizado por los propios equipos o incluso por la empresa responsable de la retransmisión, en las que se compite contra un número mayor de adversarios 
y las recompensas son mayores y exclusivas (González, 2020). Este tipo de aplicaciones vienen influenciadas por otras que anteriormente se han utilizado en los deportes tradicionales. Son muy conocidas, por ejemplo, el 'As Biwenger' perteneciente al diario de información deportiva As o 'Fantasy', el servicio de ligas virtuales del periódico Marca.

Como generadores de experiencias, los medios digitales no están sujetos únicamente a la libertad de uso - lúdica o no- del usuario, sino que, a partir de su naturaleza cultural, favorecen la creación personal como resultado de las interacciones llevadas a cabo por cada sujeto (Bourdieu, 2018). Los ejemplos mencionados hasta aquí ponen de relieve el empleo de una novedosa ludonarrativa para reforzar la relación entre el usuario, el videojuego y el deporte electrónico, y cuyo resultado se traduce en distintas recompensas para el jugador. Sin embargo, esta correspondencia también puede dirigirse hacia una influencia en el desarrollo del relato a partir de los eventos temáticos. En 2017, durante la final del mundial de League of Legends celebrada en China, la ceremonia inaugural consistió en una representación del videojuego en la realidad, introduciendo a los bailarines y a los cantantes en la representación. Mientras el grupo de música Against The Current entonaba el Legends never die, himno correspondiente al mundial de ese año, uno de los dragones que aparece en el videojuego - y uno de los objetivos de los jugadores en las partidasirrumpió en las gradas del estadio gracias a la realidad virtual (González, 2017).

El éxito de la incursión de un elemento virtual en una emisión televisada en directo en distribución broadcast y también streaming —un total de treinta canales y plataformas en diecinueve idiomas-motivó que la empresa diseñadora del videojuego, Riot Games, replicase la misma técnica en ulteriores ceremonias de apertura, pero con progresivas mejoras del contenido. Las finales del año 2018 tuvieron lugar en el estadio de Munhak (Corea del Sur), y la realidad virtual fue protagonista de nuevo en el acto de apertura. Aquí, sin embargo, la preparación del espectáculo tuvo lugar meses antes y comenzó con un gran evento en el videojuego llamado 'KDA' que tenía que ver con cuatro de los personajes del videojuego. A través de distintas misiones, se podían desbloquear nuevas funcionalidades en la historia. Estos cuatro personajes constituirían la piedra angular de la ceremonia de apertura: junto a las cantantes Soyeon, Madison Beer, Jaira Burns y Miyeon, del célebre grupo de K-pop (G)I-del, los personajes del videojuego introducidos mediante realidad virtual - Ahri, Evelynn, Kai'sa y Akali- hacían las veces de las cantantes, en un espectáculo que logró captar una de las mayores audiencias acumuladas en el mundo. Solo esa canción superó los doscientos cuarenta millones de reproducciones en todos los canales, cien millones en YouTube (Pérez Irigoyen, 2018). 
Imagen 4. Final Mundial League of Legends 2018. Ceremonia de apertura

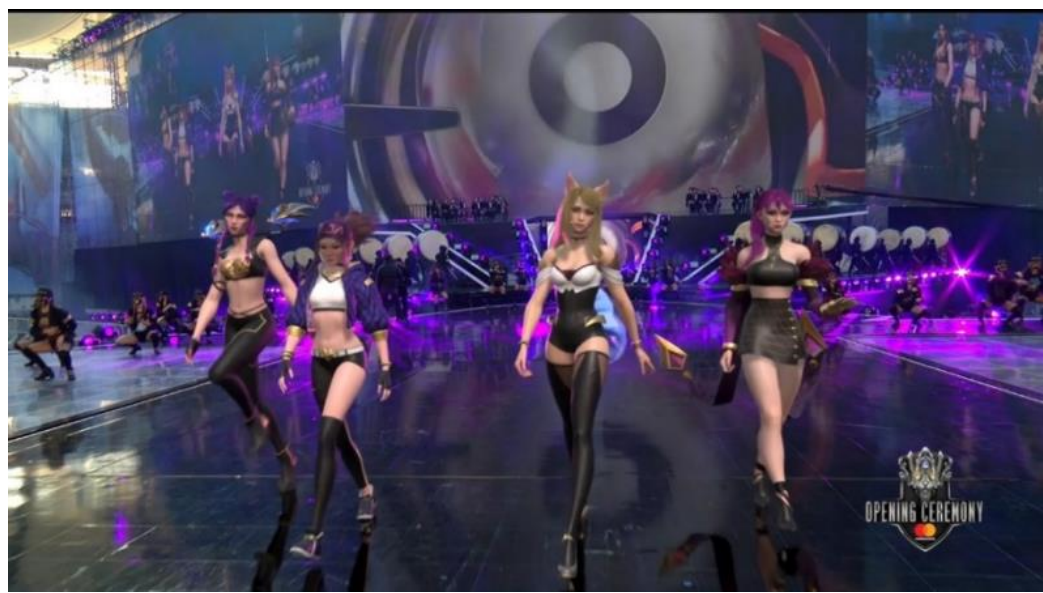

Fuente: Dessignare.com.

Al año siguiente, Riot Games siguió invirtiendo esfuerzos en la mejora de la realidad virtual como eje central de la ceremonia de apertura. El evento sobre el que versaría la final del año 2019 se llamó 'True Damage', e involucraba a otros personajes del videojuego distintos a los del anterior año, aunque con una idea parecida. Los cantantes este año fueron Thutmose, Duckwrth, Soyeon y la mundialmente conocida Becky G. Los cuatro tenían su contraparte digital en personajes que se involucraban en la ceremonia y daban al espectador una sensación de inmersión total.

En 2020, los aficionados a los deportes electrónicos en general, y al League of Legends en particular, tenían las expectativas muy altas con el mundial previsto para el mes de octubre, pues varios aspectos hacían presagiar que se trataría de una cita única: el videojuego cumplía su décimo aniversario y desde Riot Games se habían anunciado muchas sorpresas para el espectador. El mundial se había trasladado a China, donde el juego tiene el mayor número de seguidores y la infraestructura permitía introducir novedosas herramientas tecnológicas. Además, en los meses previos a la celebración del Mundial de League of Legends de 2020, la empresa había lanzado un nuevo evento temático en el videojuego denominado 'Florecer espiritual' (League of Legends, 2020), que consistía en la presentación de nuevos personajes para el videojuego, la introducción de funcionalidades añadidas para algunos de los protagonistas y una narrativa más amplia basada en la lógica de la recompensa: según el usuario vaya jugando en distintas partidas, el jugador irá desbloqueando conversaciones con los personajes protagonistas del evento, en el que irá 
aprendiendo narrativa del videojuego y del evento 'Florecer espiritual' (Guiñón, 2020).

\section{Imagen 5. Personajes del League of Legends con la temática 'Florecer espiritual'}
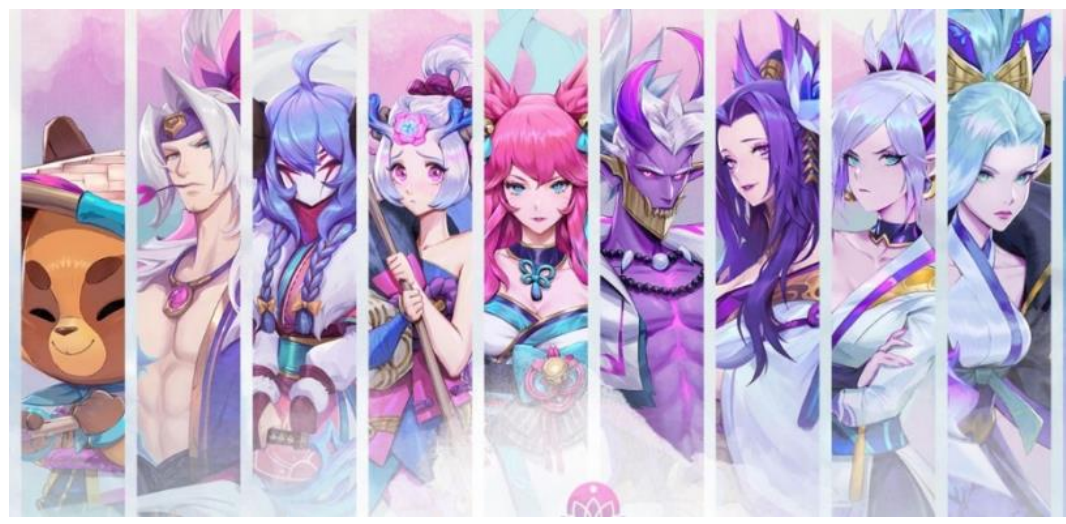

Fuente: Movistar eSports.

A pesar de ello, la llegada del coronavirus, y el posterior confinamiento sanitario en prácticamente todo el mundo, trastocó los planes de la empresa para la final del Mundial 2020. Los distintos lugares para la celebración de las fases previas, la inclusión del público en las partidas y la celebración de un gran espectáculo de masas fueron sustituidos por una gran 'burbuja' libre de coronavirus en la que se concentraba a los jugadores y al cuerpo técnico que participaban en el evento. Por lo tanto, todos los planes de Riot Games -incluso para vertebrar la temática del evento-fueron desechados. Finalmente, la empresa diseñadora del videojuego organizó la ceremonia de apertura haciendo un paseo a través de los diez años que ha cumplido el videojuego en 2020, con los himnos de años pasados, y haciendo especial hincapié en una de las temáticas ya realizadas por la compañía: 'KDA' con los personajes del videojuego - Ahri, Kai'sa, Evelyn y Akali- llevaron el peso de la ceremonia de apertura a través de la canción de 2020 Take over.

Pese a no poder organizar la ceremonia de apertura como les hubiera gustado debido a las complicaciones de la COVID-19, la experiencia sirvió para evidenciar otro ejemplo de cómo un deporte electrónico - y por tanto sus espectadores y seguidores-pueden influir en la narrativa del videojuego. « $\mathrm{Si}$ el medio permite desarrollar contenidos, incorporar a diferentes públicos y ofrecer nuevas experiencias, entonces el producto es completo en su transmedialidad, pues la experiencia del usuario resulta ampliada dentro del mismo universo narrativo» (Alonso Martínez et al., 2020: 186.). La estrategia 
de introducir un recurso narrativo previo que permita al jugador desbloquear nuevas misiones e historias en un videojuego conducentes a los eventos de League of Legends, servirá para estimular a los jugadores habituales y también para sumar nuevos seguidores al que hoy se constituye como el $e$-sport más popular del mundo.

\title{
6. Discusión y conclusiones
}

Frente a las narrativas propias de los videojuegos, cuyas variables formales, expresivas y técnicas están orientadas al disfrute individual de los jugadores, el planteamiento narrativo de los e-sports se dirige a la participación directa y simultánea de los usuarios en su condición de jugadores y de espectadores con el objetivo de que esta segunda faceta incentive el rol de la primera. Se amplían, así, las dinámicas de aprendizaje, experimentación y dominio de habilidades que caracterizaron a los deportes electrónicos en su desarrollo inicial, y se propicia la integración de las interacciones y emociones colectivas sobre los estímulos de diversión, atención y autocontrol individual que se corresponden con la naturaleza de los videojuegos tradicionales:

\begin{abstract}
Desde el punto de vista intelectual, la complejidad de la mayor parte de los juegos actuales de ordenador permite desarrollar no solo aspectos motrices sino, sobre todo, procedimientos tales como las habilidades para la resolución de problemas, la toma de decisiones, la búsqueda de información o la organización, etc. Desde el punto de vista afectivo, los juegos ejercen una importante motivación y pueden utilizarse para el trabajo de aspectos relativos a la autoestima (Gros Salvat, 2006: 8).
\end{abstract}

Aunque en League of Legends se han identificado sugerentes y renovadas técnicas para potenciar la narrativa de los videojuegos con categoría de $e$-sport, cabe apuntar que aún podrían tener mayor relevancia. Como productos digitales que requieren de la interacción de los usuarios para definir algunas de sus características o resultados, uno de los aspectos que se deben potenciar es el feedback con las informaciones y opiniones: aunque se trata de una herramienta simple, los videojuegos la utilizan ya como respuesta ante las acciones de un personaje (Calvo Sastre, 1998). Además, los portales más populares recurren a las puntuaciones para calificar los nuevos títulos a partir de los votos que ofrecen los usuarios. En un escenario en el que los deportes electrónicos siguen creciendo, lo cual repercute en sus propias narrativas, el uso del feedback en los grandes torneos de videojuegos ha de servir para modificar, cambiar, ajustar e incluso incorporar nuevas narrativas.

También se podría potenciar la relación entre usuario, videojuego y deporte electrónico otorgando más importancia a la visualización de los e-sports; no solo con grandes eventos que unan los tres elementos de la relación, sino 
tratando de que la narrativa del videojuego se corresponda con su visualización; por ejemplo, con la inclusión de recompensas encaminadas al desbloqueo de historias y nuevos espacios necesarios para completar el reto. Ante la creciente penetración social de los deportes electrónicos, el abanico de posibilidades que se abre en torno al mundo de los videojuegos parece inimaginable, sobre todo con el avance de las tecnologías de conexión. El 5G, por ejemplo, puede mejorar la velocidad de acceso, permitir a más usuarios estar conectados a un mismo servicio de streaming, aumentar la calidad de la imagen... La realidad virtual está llamada a incrementar la inmersión del usuario en los mundos ficticios y a crear nuevas sensaciones situando al jugador dentro del propio videojuego. Incluso la inteligencia artificial puede resultar de gran ayuda para el sector, identificando nuevos ángulos de cámara y de seguimiento de la acción en los torneos y midiendo las aptitudes de cada usuario con el fin de que sepa cómo mejorar (Mateo, 2019). Un horizonte que no solo logrará consolidar las particularidades narrativas de los e-sports, sino que multiplicará su potencial como propuesta digital cada vez más propicia para la hibridación de juego, deporte y espectáculo.

\section{Referencias bibliográficas}

Alonso MarTínez, D., NAVAZo-OSTÚA, P., \& PÉREZ-Escolar, M. (2020). Narrativa periodística en videojuegos. Estudio de caso del serious game 'The Republia Times.' Miguel Hernández Communication Journal, 11. https: / / tinyurl.com/525dvaxf

ANTón, M. (2020). eSports: El efecto del coronavirus en el mundo del streaming | Marca.com. Diario Marca. https://tinyurl.com/3rydx97x

ASOCIACIÓN ESPAÑOLA DE VIDEOJUEGOS. (2019). La industria del videojuego en

España. https: //tinyurl.com/y7s82 mpz

BERZOSA, M. (2017). Youtubers y otras especies.

https: //tinyurl.com/nfmw9rzv

BouRdiEU, P. (2018). Las estrategias de la reproducción social. Siglo veintiuno.

CAlVo SASTRE, A. M. (1998). Videojuegos: del juego al medio didáctico. Quaderns Digitals. https: //tinyurl.com/2bcp838c

CARrillo Vera, J. A. (2016). La dimensión social de los videojuegos "online": de las comunidades de jugadores a los "e-Sports." Index.Comunicación. Revista Científica de Comunicación Aplicada. Universidad Rey Juan Carlos., 5(1), 39-51. https: //tinyurl.com/ynvx8z2b

DíAZ, C. (2020). Drops League of Legends: cómo conseguir drops en LoL viendo Lec y Lcs - Movistar eSports. Diario AS.

https: //tinyurl.com/3m5 $2 \mathbf{2 r n j}$ 
Documania. (2014). La Guerra Fría 8 - Sputnik (1949-1961) - YouTube. https://tinyurl.com/mc8zc98j

ESPORTS CHARTS. (2018). Worlds 2018 - 200 million viewers at once - Esports Charts. Esports Charts. https: //tinyurl.com/9szxp37v

EUROPA PRESS. (2019). Los servidores más cercanos y el "streaming", principales claves del impacto del videojuego en el tráfico de Internet. Europa Press. https: //tinyurl.com/69fy9wxs

GARCíA, F. (2019). Todo lo que necesita saber sobre el programa de Recompensas de Visionado de League of Legends. Dexerto. https: //tinyurl.com/3v5a2588

GoNZÁLEZ, S. (2017). Worlds 2017: Así ha sido la espectacular ceremonia de clausura... ¡ con un dragón virtual! - Vandal Sports. Vandal Sports. https: / / tinyurl.com/y27fbna6

GonZÁLEZ, S. (2020). Lvp y Riot lanzan Lec SuperFantasy, el nuevo juego para aderezar la LEC - Vandal Sports. Vandal Sports. https: / / tinyurl.com/2u4xef $\mathbf{j}$

Gros SAlVAT, B. (2006). La dimensión socioeducativa de los videojuegos. Edutec. Revista Electrónica de Tecnología Educativa, 12. https://doi.org/10.21556/edutec.2000.12.557

GuiÑón, Á. (2020). Todos los detalles de Spirit Blossom (Florecer Espiritual), el nuevo evento de LoL - Movistar eSports. Diario AS. https: //tinyurl.com/fjvjksvy

HAMMERSLEY, M. (1986). Case studies in classroom research. Open University Press.

HeRnándeZ-SAmpieri, R., Fernández Collado, C., \& LuCio BAPTiSTa, M. del P. (2014). Metodología de la investigación. McGraw Hill.

ISFE. (2020). Key facts 2020. https: / / tinyurl.com/nfon37e3

IWERKS, L. (2019). League of Legends: Orígenes. Netflix. https: / / tinyurl.com/due64cxd

KENT, S. L. (2001). The ultimate history of video games : from Pong to Pokémon and beyond : the story behind the craze that touched our lives and changed the world. Prima Pub. https://tinyurl.com/sherueja

LEAGUE OF LEGENDS. (2020). Florecer Espiritual - League of Legends. League of Legends. https://tinyurl.com/cm3carmt

LEWIS, L. (2020). Infographic: What Happens In An Internet Minute 2020 by Lori Lewis | Merge | AllAccess.com. All Access. https: //tinyurl.com/3jz3jcv3

Liga DE VIDEOJUEGOS PROFESIONAL. (2020). Juega a SuperFantasy, el fantasy oficial de la Superliga Orange. Liga de Videojuegos Profesional. https: //tinyurl.com/8sftcedm 
LOLESPORTS. (2019). 2019 World Championship Hits Record Viewership. https://tinyurl.com/5ya5rft3

LÓPEZ-BARAJAS, E., \& MontoyA, J. M. (1995). El estudio de casos. Fundamentos y metodología. Uned.

MARCA ESPORTS. (2019). League of Legends: Más de 8 millones de jugadores juegan diariamente a League of Legends | Marca.com. Diario Marca. https: //tinyurl.com/4ebayh35

MARTín MuÑoZ, D., \& PEDRERo EsteBAN, L. M. (2019). Esports: Origen, evolución y tendencias. Vista. Revista de Cultural Visual, 4. https: //tinyurl.com/y3mv9ktw

MATEO, J. Á. (2019). El 5G y la inteligencia artificial, a debate en el futuro de los esports. Xataka Esports. https: //tinyurl.com/5f53en3a

MElLADO, J. (2018). Worlds 2018: ¡Récord de audiencia en la historia del League of Legends! Esportmaníacos. https: //tinyurl.com/3ju6daz7

NEIRA, E. (2020). Streaming Wars. La nueva televisión. Cúpula.

NEWZOO. (2020). Global Esports Market Report Table of Contents. https: //tinyurl.com/rz2nfj7e

PÉrez IRIGOYEN, D. J. (2018). La Apertura de las Finales del Mundial de League of Legends fue uno de los eventos más vistos del mundo - Dessignare Media - Arte Visual, Diseño y Animación. Dessignare. https: / / tinyurl.com/2tybwy43

QuiroGA, J. M. (2018, November). Primeros desarrollos de tecnología radar en los principales beligerantes de la II Guerra Mundial. Un análisis desde la perspectiva Ciencia, Tecnología y Sociedad. Ciencia, Docencia y Tecbnología, 29, 43-45. https: //tinyurl.com/fyumbbcw

SCOLARI, C. A. (2013). Homo videoludens 2.0 De Pacman a la gamifi cation Nueva edición ampliada. https: //tinyurl.com/24hcv7cd

TAYLOR, T. L. (2015). Raising the Stakes. E-Sports and the Professionalization of Computer Gaming. The MIT Press.

TAYLOR, T. L. (2018). Watch Me Play: Twitch and the Rise of Game Live Streaming. Princeton University Press.

VELASCO, J. J. (2011). Historia de la tecnología: Computer Space, los orígenes de Atari. Hipertextual. https: //tinyurl.com/9kmsx87d

YIN, R. K. (1989). Case Study Research. Design and Methods. Sage. 